\title{
Transcatheter Arterial Embolization Using Microspheres for Palliating Pain from Bone Metastasis in a Patient with Cholangiocellular Carcinoma
}

\author{
Shinichi Morita ${ }^{1}$, Shunsuke Sugawara ${ }^{2}$, Takeshi Suda ${ }^{1}$, Didik Prasetyo ${ }^{1}$, Takahiro Hoshi ${ }^{1}$, \\ Satoshi Abe ${ }^{1}$, Kazuyoshi Yagi ${ }^{1}$ and Shuji Terai ${ }^{3}$
}

\begin{abstract}
:
A 72-year-old man with intractable left shoulder pain due to bone metastasis from cholangiocellular carcinoma was admitted to our hospital. Computed tomography showed an osteoblastic metastatic lesion of the left scapula. Since the pain persisted even after the administration of opioids and external irradiation, microspheres were injected through a catheter; the catheter tip was placed at the arteries feeding the metastatic lesion. After the intervention, the shoulder pain was alleviated without any procedure-related complications, leading to a reduction in the opioid dose. This case suggests the efficacy of transcatheter arterial embolization using microspheres for palliating pain from bone metastasis.
\end{abstract}

Key words: transcatheter arterial embolization, microsphere, bone metastasis, cholangiocellular carcinoma

(Intern Med 60: 241-246, 2021)

(DOI: 10.2169/internalmedicine.5351-20)

\section{Introduction}

In recent years, the number of patients with bone metastases has increased due to the increase in the number of cancer patients and their prolonged prognoses due to advances in cancer treatment $(1,2)$. Bone metastases are often asymptomatic in the early stages (3), but as they progress, they cause pain, spinal cord compression, and pathological fractures resulting in dyskinesias, paralysis, and a significantly decreased quality of life (QOL) (4-8). Pain is the most frequent symptom of bone metastasis. Half of the cancer pain cases have been attributed to bone metastases (4). Therefore, it is important to properly control pain in patients with bone metastasis.

There are several standard treatments for metastatic bone pain, including medication (9-11), radiation therapy $(12,13)$, and surgery (14). Each treatment has been reported to have high efficacy, and patients can receive multiple treatment types in combination. However, $20-40 \%$ of patients with bone metastasis receiving multiple standard treatments reportedly experience difficulty with pain control (4).

In recent years, several reports have indicated that transcatheter arterial embolization (TAE) is an effective treatment method for metastatic bone pain (15-19). We herein report a case of cholangiocellular carcinoma with bone metastasis-induced pain that was refractory to standard treatment methods but rapidly relieved by TAE.

\section{Case Report}

This study was conducted according to the Declaration of Helsinki. It was approved by our institution's review board, and the patient provided his consent for the specific treatment and publication of the results.

A 72-year-old Japanese man was referred to our hospital due to severe pain in his left shoulder. He had been diagnosed with cholangiocellular carcinoma eight months earlier (Fig. 1A). A large tumor occupying the left lobe of the liver was observed, and dynamic contrast-enhanced computed to-

${ }^{1}$ Departments of Gastroenterology and Hepatology, Uonuma Institute of Community Medicine Niigata University Hospital, Japan, ${ }^{2}$ Department of Diagnostic Radiology, National Cancer Center Hospital, Japan and ${ }^{3}$ Division of Gastroenterology and Hepatology, Graduate School of Medical and Dental Sciences, Niigata University, Japan

Received: May 15, 2020; Accepted: July 20, 2020; Advance Publication by J-STAGE: September 12, 2020

Correspondence to Dr. Shinichi Morita, m0riz0u@extra.ocn.ne.jp 

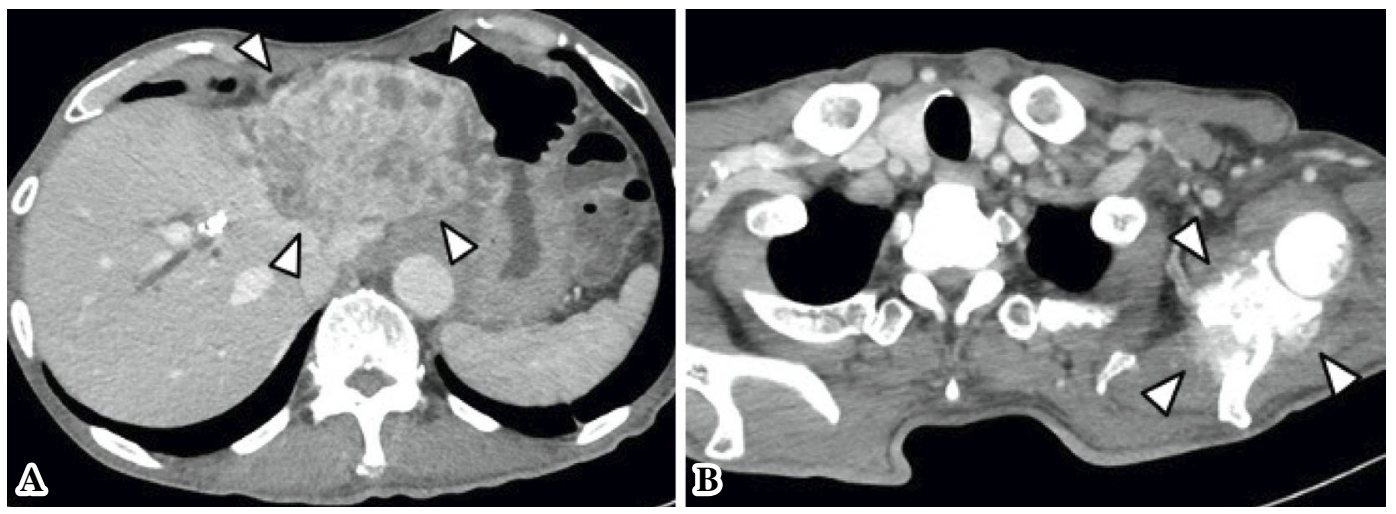

Figure 1. Abdominal and chest computed tomography images. a: A well-enhanced cholangiocellular carcinoma is located in the left hepatic lobe (arrowheads). b: Osteoblastic bone metastasis is present in the left scapula bone (arrowheads).

Table. Results of Laboratory Investigation.

\begin{tabular}{cclrlr}
\hline Hematologic test & & Chemistry & & BUN & $16.9 \mathrm{mg} / \mathrm{dL}$ \\
White blood cells & $4,900 / \mu \mathrm{L}$ & Total protein & $6.8 \mathrm{~g} / \mathrm{dL}$ & Creatinine & $0.7 \mathrm{mg} / \mathrm{dL}$ \\
Neutrophils & $73.6 \%$ & Albumin & $3.4 \mathrm{~g} / \mathrm{dL}$ & Sodium & $135 \mathrm{mmol} / \mathrm{L}$ \\
Lymphocytes & $20.3 \%$ & AST & $37 \mathrm{IU} / \mathrm{L}$ & Potassium & $4.5 \mathrm{mmol} / \mathrm{L}$ \\
Monocytes & $5.1 \%$ & ALT & $28 \mathrm{IU} / \mathrm{L}$ & Chloride & $98 \mathrm{mmol} / \mathrm{L}$ \\
Eosinophils & $0.8 \%$ & ALP & $1,551 \mathrm{IU} / \mathrm{L}$ & CRP & $3.15 \mathrm{mg} / \mathrm{dL}$ \\
Basophils & $0.2 \%$ & $\gamma$-GTP & $125 \mathrm{IU} / \mathrm{L}$ & CEA & $9.1 \mathrm{ng} / \mathrm{mL}$ \\
Red blood cells & $375 \times 10^{4} / \mu \mathrm{L}$ & T.Bil & $0.7 \mathrm{mg} / \mathrm{dL}$ & CA19-9 & $1,849 \mathrm{U} / \mathrm{mL}$ \\
Hemoglobin & $11.4 \mathrm{~g} / \mathrm{dL}$ & D.Bil & $0.2 \mathrm{mg} / \mathrm{dL}$ & AFP & $3.5 \mathrm{ng} / \mathrm{mL}$ \\
Platelet count & $31.5 \times 10^{4} / \mu \mathrm{L}$ & LDH & $141 \mathrm{IU} / \mathrm{L}$ & PIVKA-II & $12 \mathrm{mAU} / \mathrm{mL}$ \\
\hline
\end{tabular}

AST: aspartate aminotransferase, ALT: alanine aminotransferase, ALP: alkaline phosphatase, $\gamma$-GTP: $\gamma$-glutamyl transpeptidase, T.Bil: total bilirubin, D.Bil: direct bilirubin, LDH: lactate dehydrogenase, BUN: blood urea nitrogen, CRP: C-reactive protein, CEA: carcinoembryonic antigen, CA19-9: carbohydrate antigen 19-9, AFP: $\alpha$-fetoprotein, PIVKA-II: protein induced by vitamin $\mathrm{K}$ absence or antagonist-II

mography (CT) showed that the whole tumor was gradually well enhanced. Therefore, tumor needle biopsies were performed to distinguish it from hepatocellular carcinoma, and a pathological diagnosis of cholangiocellular carcinoma was established.

He was performed systemic chemotherapy involving the combination administration of gemcitabine and cisplatin as the first treatment and tegaful/gimeracil/oteracil as the second treatment and then discontinued chemotherapy one month before referral our hospital due to the progression of the disease. He was receiving supportive care at the time of his presentation to our hospital. Laboratory tests during admission revealed increased levels of alkaline phosphatase (ALP, 1,551 IU/L). Regarding the tumor markers, carbohydrate antigen 19-9 (CA19-9, 1,849 U/mL) was remarkably increased, whereas normal levels of $\alpha$-fetoprotein (AFP, 3.5 $\mathrm{ng} / \mathrm{mL}$ ) and protein induced by vitamin $\mathrm{K}$ absence or antagonist-II (PIVKA-II, $12 \mathrm{mAU} / \mathrm{mL}$ ) were observed (Table).

CT of the left shoulder revealed an osteoblastic metastatic lesion of the left scapula (Fig. 1B). He was administered bisphosphonates, oral morphine, and external irradiation (20 Gy), but the pain persisted with a score of $7 / 10$ on the numerical rating scale (NRS). The dose of morphine was in- creased to $120 \mathrm{mg} /$ day as needed for severe pain, but the patient experienced drowsiness that prohibited further increases in the morphine dose. The patient's Eastern Cooperative Oncology Group Performance Status worsened from 1 to 3 due to uncontrollable pain and drowsiness from the morphine. Therefore, TAE was performed to control the left shoulder pain because the tumor was well-enhanced by contrast CT.

With the informed consent of the patient, TAE was performed via a left radial artery approach. Left brachial artery angiography showed a tumor stain from the thoracoacrominal artery and the circumflex scapular artery (Fig. 2A-C). A microcatheter (Sniper 2- $\mu$ 7; Terumo Clinical Supply, Tokyo, Japan) with a 2.0-F tip was inserted into these arteries, and TAE was performed with microspheres (Embosphere; Nippon Kayaku, Tokyo, Japan) ranging from 300 to $500 \mu \mathrm{m}$ in diameter. Subsequent angiography performed immediately after the TAE procedure revealed the almost complete disappearance of the tumor stain (Fig. 2D). No adverse events were observed during the procedure. Although the patient experienced transient pain [Numerical Rating Scale (NRS) score 8/10] with mild pyrexia requiring additional administration of analgesics for a couple of days due to postembolism syndrome, the pain gradually improved in the following week (NRS score 3/10) resulting in the reduction of 


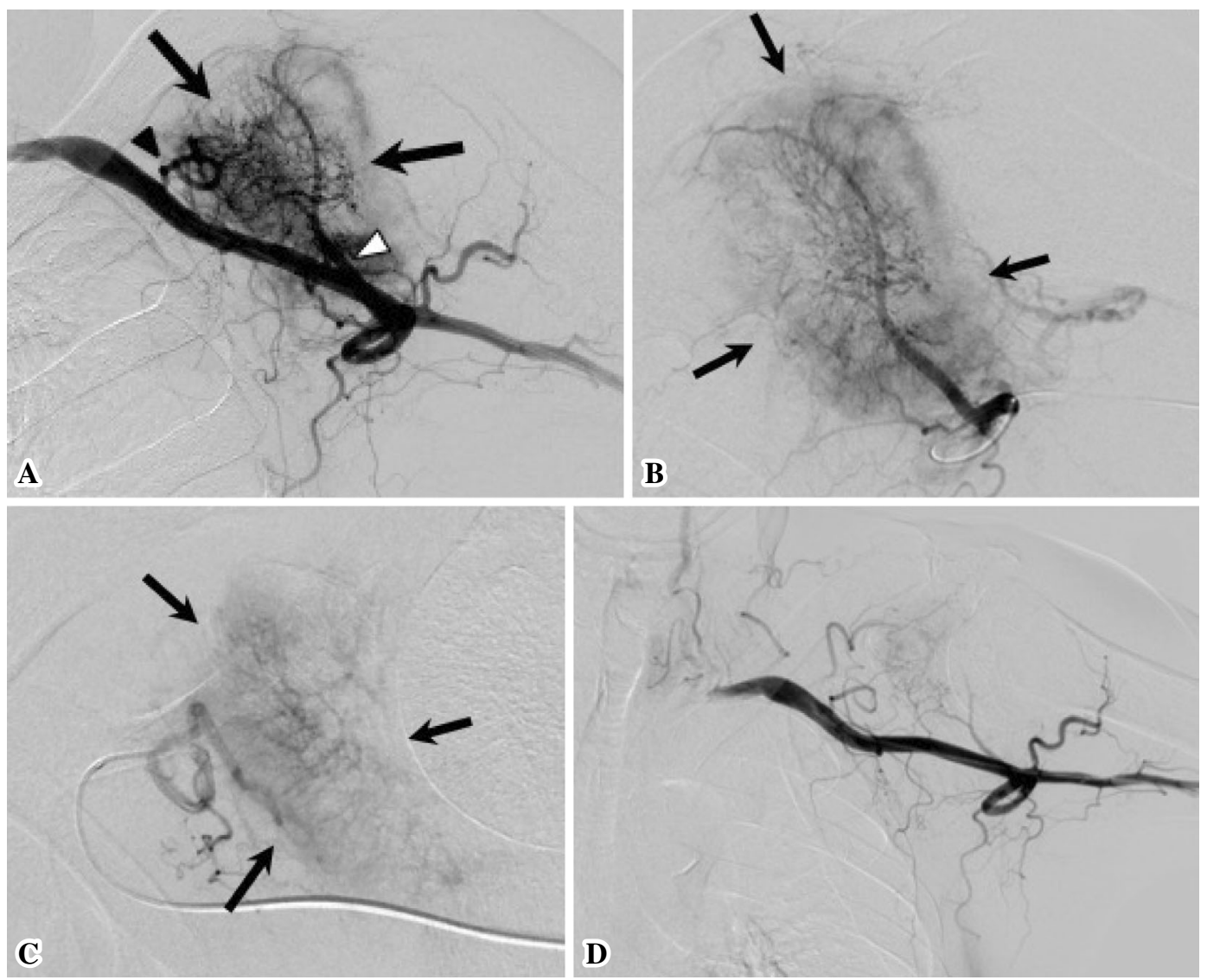

Figure 2. Angiography images. a: Angiography via the left brachial artery shows tumor enhancement (arrows). The tumor is mainly fed by the circumflex scapular artery (white arrowhead) and the thoracoacromial artery (black arrowhead). b: Selective angiography via the circumflex scapular artery demonstrates prominent tumor enhancement (arrows). c: Selective angiography via the thoracoacromial artery demonstrates prominent tumor enhancement (arrows). d: Angiography via the left brachial artery immediately after TAE shows the nearly complete disappearance of tumor enhancement. Arterial depiction, showing that the circumflex scapular artery and the thoracoacromial artery are well preserved.

the morphine dose to $90 \mathrm{mg} /$ day and alleviation of the drowsiness.

Three months later, the patient died due to the progression of the primary disease. Shortly before his death, his shoulder pain was managed with rescue oral morphine taken several times a day. The clinical course and changes in serum C-reactive protein and CA19-9 before and after TAE are shown in Fig. 3.

\section{Discussion}

In this case, TAE alleviated bone metastasis-induced pain that was refractory to a combination of standard treatments, including bone-modifying agents (BMAs), opioids, and external irradiation. TAE is an interventional radiologic technique in which a catheter is advanced to a target artery, and embolic materials are injected into the blood vessel to block the arterial blood flow. Preoperative TAE is a widely accepted technique for reducing blood loss during metastatic bone surgery $(20,21)$. In addition, the pain-relieving effect of TAE on bone metastases has been reported in several pi- lot studies and retrospective studies (15-19). The effective rate of pain relief ranges between $60-80 \%$, and the median time to pain alleviation is 2 days (19). The analgesic effect of TAE lasts an average of 2-8 months, and TAE can be repeated when the effect diminishes $(15,18)$. Furthermore, TAE has been reported to be effective against bone metastases of not only hypervascular tumors, such as hepatocellular carcinoma, thyroid cancer, and renal cell carcinoma, but also colorectal cancer, ovarian cancer, uterine cervical cancer, and esophageal cancer (19). This case showed a similar profile, with the pain alleviated shortly after the TAE intervention and lasting for at least three months. Injection and/or impregnation of anticancer agents can be simultaneously performed with embolization (19). Therefore, TAE is a promising option for palliating pain from bone metastasis. However, thus far, there have been no well-evidenced studies on this issue. Therefore, a further prospective evaluation of the safety and efficacy should be conducted.

The mechanism underlying the pain relief provided by TAE is unclear and likely multi-faceted. Several hypotheses have been reported (19). TAE blocks the arterial supply of 


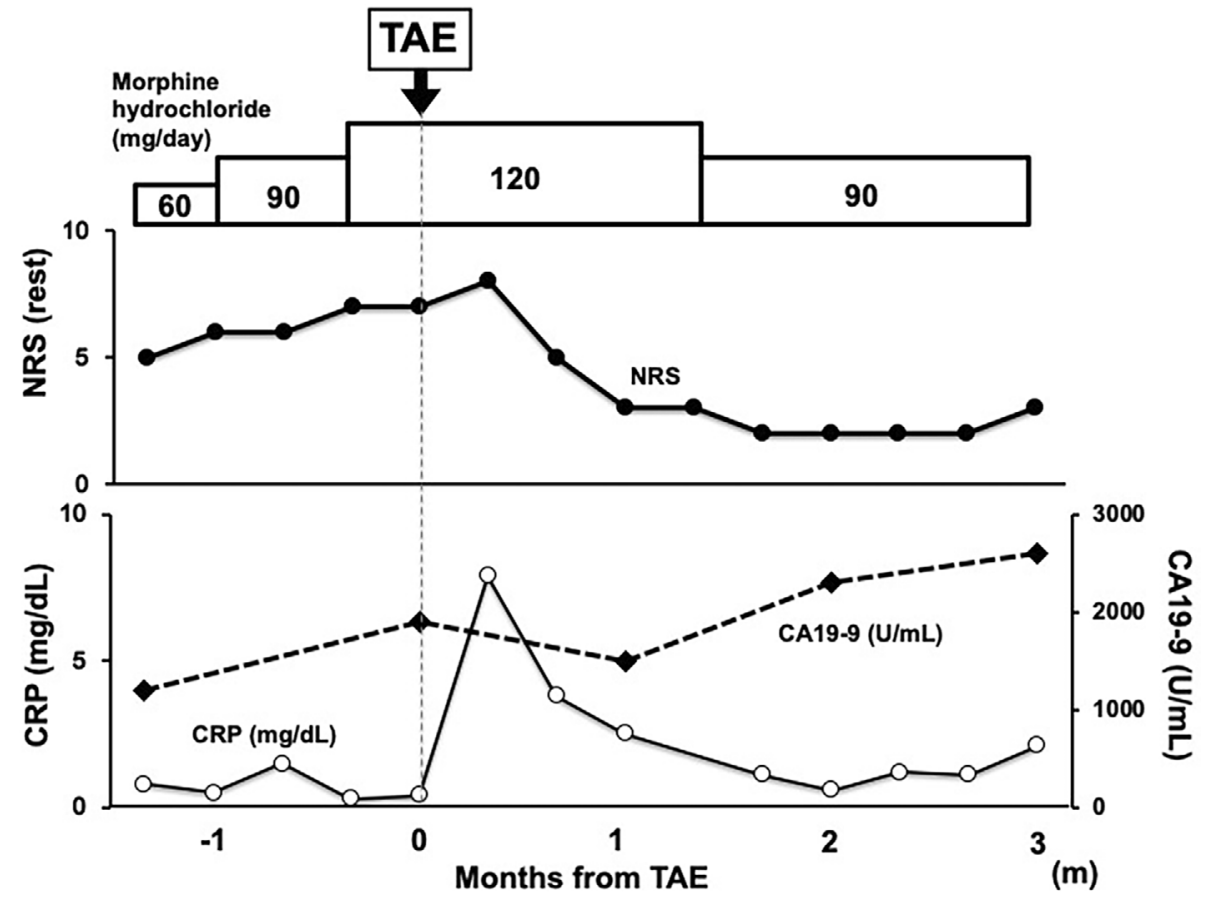

Figure 3. Clinical course of the patient before and after TAE. The time-dependent levels of the pain score with NRS are shown in the upper panel. In the lower panel, the solid line and dotted line show the serum CRP and CA19-9 levels, respectively. NRS: Numerical Rating Scale, CRP: C-reactive protein, CA19-9: carbohydrate antigen 19-9, TAE: transcatheter arterial embolization

the metastatic tumors, which is expected to slow tumor growth and reduce the tumor burden, resulting in the delay or cessation of the destruction of the periosteum. In addition, reduced blood flow and edema within the tumor may decrease the direct pressure on adjacent tissues and nerves.

The complications of TAE are typically minor and infrequent and mainly occur at the puncture site or with the use of contrast medium; the frequency is approximately $3 \%$ or $5-7 \%$, respectively (22). Approximately $10 \%$ of patients undergoing TAE experience post-embolism syndrome that induces a fever and temporary deterioration of pain (22). When embolization occurs in arteries supplying soft bone tissues, neuropathy, osteonecrosis, muscle necrosis, or dermal necrosis can occur. If the artery of Adamkiewicz, which joins the anterior spinal artery from outside of the spine, is embolized during TAE for spinal tumor, it can result in paraplegia, a loss of sensation for pain and temperature, and bladder-rectal dysfunction-so-called spinal artery syndrome (23). However, given adequate training, the procedure is sophisticated enough to avoid these complications due to the embolization of non-target arteries.

There are several types of embolic materials used in TAE. Gelatin particles are classically used for blocking the blood supply in tumors, such as in transcatheter arterial chemoembolization for hepatocellular carcinoma (24); however, microspheres have been popularized in recent years (25). Gelatin particles are temporary embolic materials, and the embolized blood vessels are recanalized within a few weeks (26). However, microspheres are spheres with a uniform particle shape comprising non-aggregating permanent embolic materials that do not cause inflammation. Possibly, a blood vessel meeting the target diameter can be embolized, resulting in less inflammation and pain after embolization. Therefore, microspheres were used in this case because we considered the technique safe and the embolic effect prolonged (27).

The particle size of the microspheres used for TAE of bone metastases is recommended to be 300-500 $\mu \mathrm{m}(20,21)$. Vessels in the bone and soft tissue areas are predominantly arterio-venous shunts, and the use of smalldiameter embolic materials increases the risk of pulmonary embolism (28). In addition, drug-eluting beads can be loaded with anticancer drugs; however, adverse events associated with the anticancer drugs may occur (29). In this case, the purpose of TAE was palliation, and TAE was performed without using an anticancer drug, considering the risk of adverse events.

The standard treatment of metastatic bone pain is performed with a combination of BMAs (9), opioids (10, 11), external irradiation $(12,13)$, and surgery (14); however, 20$40 \%$ of cancer patients are refractory to these treatments (4). The main purpose of BMAs, such as bisphosphonate and denosumab, is to reduce skeletal-related events and shorten the duration of the pain. BMA has been reported to be useful for bone metastases arising from breast cancer, prostate cancer, and digestive cancers. Although the long-term administration of BMA can prevent metastatic bone pain, it has little analgesic effect on existing pain. Opioids are highly effective and are the first choice in the treatment of cancer pain. The various dosages and forms allow for ad- 
justments depending on the patient's pain type and level. However, adverse effects occasionally limit the tolerated dose needed for sufficient pain relief. External irradiation can relieve pain and significantly improve the patients' QOL in $59-73 \%$ of the cases $(12,13)$. Although combinatorial prescription of analgesics is required until the irradiation starts showing efficacy in the several-weeks-long early treatment phase, the therapeutic effect of external irradiation lasts for approximately 30 weeks on average. Surgery is considered the salvage treatment for pathological fractures and spinal cord compression due to bone metastases. Surgery is performed for the purpose of pain relief and improvement in the QOL, considering the general condition of the patient, invasiveness of the surgery, and the patient's prognosis. Patients at the terminal stage of their disease are not often good candidates for surgery.

In conclusion, TAE is expected to achieve a relatively quick response with high efficacy and low invasiveness in the majority of patients experiencing metastatic bone pain. This case suggests that TAE performed at an early stage complements the standard treatments and improves the QOL in patients with painful bone metastasis.

This case report was approved by the institutional Human Investigation Committee of Uonuma Institute of Community Medicine Niigata University Hospital. Written informed consent was obtained from the patient in accordance with the Declaration of Helsinki for the publication of this case report.

The authors state that they have no Conflict of Interest (COI).

\section{References}

1. Coleman RE. Clinical features of metastatic bone disease and risk of skeletal morbidity. Clin Cancer Res 12: 6243s-6249s, 2006.

2. Rich SE, Chow R, Raman S, et al. Update of the systematic review of palliative radiation therapy fractionation for bone metastases. Radiother Oncol 126: 547-557, 2018.

3. Ursavas A, Karadag M, Uzaslan E, et al. Can clinical factors be determinants of bone metastases in non-small cell lung cancer? Ann Thorac Med 2: 9-13, 2007.

4. Lipton A, Theriault RL, Hortobagyi GN, et al. Pamidronate prevents skeletal complications and is effective palliative treatment in women with breast carcinoma and osteolytic bone metastases: long term follow-up of two randomized, placebo-controlled trials. Cancer 88: 1082-1090, 2000.

5. Barton MB, Dawson R, Jacob S, Currow D, Stevens G, Morgan G. Palliative radiotherapy of bone metastases: an evaluation of outcome measures. J Eval Clin Pract 7: 47-64, 2001.

6. Rosen LS, Gordon D, Tchekmedyian NS, et al. Long-term efficacy and safety of zoledronic acid in the treatment of skeletal metastases in patients with nonsmall cell lung carcinoma and other solid tumors: a randomized, Phase III, double-blind, placebocontrolled trial. Cancer 100: 2613-2621, 2004.

7. Saad F, Gleason DM, Murray R, et al. Long-term efficacy of zoledronic acid for the prevention of skeletal complications in patients with metastatic hormone-refractory prostate cancer. J Natl Cancer Inst 96: 879-882, 2004.

8. Morita S, Suda T, Oda C, et al. The value of (18)F-FDG PET in the diagnosis of intertrabecular vertebral metastasis in a small cell lung cancer patient with a high serum CEA level. Intern Med 58: 415-418, 2019.

9. Wong R, Wiffen PJ. Bisphosphonates for the relief of pain secondary to bone metastases. Cochrane Database Syst Rev. Forthcoming.

10. Mystakidou K, Katsouda E, Kouloulias V, Kouvaris J, Tsiatas M, Vlahos L. Comparison of transdermal fentanyl with codeine/ paracetamol, in combination with radiotherapy, for the management of metastatic bone pain. J Opioid Manag 1: 204-210, 2005.

11. Sima L, Fang WX, Wu XM, Li F. Efficacy of oxycodone/paracetamol for patients with bone-cancer pain: a multicenter, randomized, double-blinded, placebo-controlled trial. J Clin Pharm Ther 37: 27-31, 2012.

12. Steenland E, Leer JW, van Houwelingen $H$, et al. The effect of a single fraction compared to multiple fractions on painful bone metastases: a global analysis of the Dutch Bone Metastasis Study. Radiother Oncol 52: 101-109, 1999.

13. Chow E, Zeng L, Salvo N, Dennis K, Tsao M, Lutz S. Update on the systematic review of palliative radiotherapy trials for bone metastases. Clin Oncol 24: 112-124, 2012.

14. Kaloostian PE, Yurter A, Zadnik PL, Sciubba DM, Gokaslan ZL. Current paradigms for metastatic spinal disease: an evidence-based review. Ann Surg Oncol 21: 248-262, 2014.

15. Barton PP, Waneck RE, Karnel FJ, Ritschl P, Kramer J, Lechner GL. Embolization of bone metastases. J Vasc Interv Radiol 7: 8188, 1996.

16. Munk PL, Torreggiani W, Legiehn G, Morris DC, Ho SG. Palliative treatment of metastatic bone pain by embolization. Clin Radiol 56: 339, 2001.

17. Eustatia-Rutten CFA, Romijn JA, Guijt MJ, et al. Outcome of palliative embolization of bone metastases in differentiated thyroid carcinoma. J Clin Endocrinol Metab 88: 3184-3189, 2003.

18. Marciel AM, Van Zandt BL, Baxter AJ. Transcatheter arterial embolization for the palliation of painful bone lesions. Tech Vasc Interv Radiol 14: 141-149, 2011.

19. Koike $Y$, Takizawa $K$, Ogawa $Y$, et al. Transcatheter arterial chemoembolization (TACE) or embolization (TAE) for symptomatic bone metastases as a palliative treatment. Cardiovasc Intervent Radiol 34: 793-801, 2011.

20. Chatziioannou AN, Johnson ME, Pneumaticos SG, Lawrence DD, Carrasco CH. Preoperative embolization of bone metastases from renal cell carcinoma. Euro Radiol 10: 593-596, 2000.

21. Wirbel RJ, Roth R, Schulte M, Kramann B, Mutschler W. Preoperative embolization in spinal and pelvic metastases. J Orthop Sci 10: 253-257, 2005.

22. Gaba RC, Lokken RP, Hickey RM, et al. Quality improvement guidelines for transarterial chemoembolization and embolization of hepatic malignancy. J Vasc Interv Radiol 28: 1210-1223, e1213, 2017.

23. Taterra D, Skinningsrud B, Pekala PA, et al. Artery of Adamkiewicz: a meta-analysis of anatomical characteristics. Neuroradiology 61: 869-880, 2019.

24. Ikeda M, Arai Y, Park SJ, et al. Prospective study of transcatheter arterial chemoembolization for unresectable hepatocellular carcinoma: an Asian cooperative study between Japan and Korea. J Vasc Interv Radiol 24: 490-500, 2013.

25. Lammer J, Malagari $\mathrm{K}, \operatorname{Vog} \mathrm{T}$, et al. Prospective randomized study of doxorubicin-eluting-bead embolization in the treatment of hepatocellular carcinoma: results of the PRECISION V study. Cardiovasc Intervent Radiol 33: 41-52, 2010.

26. Sone M, Osuga K, Shimazu K, et al. Porous gelatin particles for uterine artery embolization: an experimental study of intra-arterial distribution, uterine necrosis, and inflammation in a porcine model. Cardiovasc Intervent Radiol 33: 1001-1008, 2010.

27. Maluccio MA, Covey AM, Porat LB, et al. Transcatheter arterial embolization with only particles for the treatment of unresectable 
hepatocellular carcinoma. J Vasc Interv Radiol 19: 862-869, 2008.

28. Brown KT. Fatal pulmonary complications after arterial embolization with 40-120- micro m tris-acryl gelatin microspheres. J Vasc Interv Radiol 15: 197-200, 2004.

29. Kim YW, Kwon JH, Nam SW, et al. Sustained multiple organ ischaemia after transarterial chemoembolization with drug-eluting beads for hepatocellular carcinoma. Exp Ther Med 15: 1479-1483,
2018.

The Internal Medicine is an Open Access journal distributed under the Creative Commons Attribution-NonCommercial-NoDerivatives 4.0 International License. To view the details of this license, please visit (https://creativecommons.org/licenses/ by-nc-nd/4.0/).

(C) 2021 The Japanese Society of Internal Medicine

Intern Med 60: 241-246, 2021 